\title{
Mike Preuss: Multimodal optimization by means of evolutionary algorithms
}

\author{
Springer, 2015, 175 pp, ISBN: 978-3-319-07407-8
}

\author{
Nailah Al-Madi ${ }^{1}$
}

Published online: 25 June 2016

(C) Springer Science+Business Media New York 2016

Dr. Mike Preuss is a leading figure in the application of computational and artificial intelligence. He has published in leading journals and conferences on various aspects of computational intelligence; in particular evolutionary computing, heuristics, search and multicriteria optimization, and served on many academic conference committees and journal editorial boards in this field.

Dr. Preuss in this book aims to shape the multimodal optimization field by bringing all its roots (i.e. niching, parallel evolutionary algorithms, and global optimization) together and collecting use cases, algorithms, and performance measures. It consists of six main chapters, plus a seventh that summarizes the book and discusses how well it has achieved its objectives.

The first chapter introduces the basics of evolutionary computations such as; optimization, objective function, global optimum, local optimality, evolutionary multimodal optimization and basins of attraction (which means partitioning the search space into portions each containing set of points that share the same local optima). It also states the main objectives of the book. Then, in chapter 2 , he discusses experimentation in evolutionary computation and its documentation, and recommends reporting rules that can speedup writing experiments and guides the readers through structured reports. Moreover, he discusses the effect of setting the optimization algorithm parameters on its performance, and the difference between controlling these parameters (parameter adaptation during the run of an optimization algorithm) and parameters tuning (improving parameter settings before the run).

In Chapter 3, he discusses niching in EC, its definitions in the literature, compares it with local search and then proposes a simple niching model. Simulated

Nailah Al-Madi

n.madi@psut.edu.jo

1 Computer Science Department, Princess Sumaya University for Technology, Amman, Jordan 
results are presented comparing equal sized basins of attraction and un-equal sized basins, showing the effect of basins size on niching algorithms which are often used in multimodal optimization. Chapter 3 assumes the existence of efficient methods for basin identification and recognition (this is discussed in next chapter). Chapter 4 investigates the Nearest-Better Clustering method (NBC) and its extensions for basin identification. Moreover, it suggests and evaluates a correction of it for large samples, and performs a large scale parameter investigation of the NBC in order to derive good default values for various settings.

Chapter 5 starts with discussing the relationship between niching and multimodal optimization and then defining four use cases for the optimization of multimodal problems, depending on the desired solution type (All-Global, All-Known, GoodSubset, and One-Global). Then, it discusses the available performance measures for multimodal optimization. At the end of this chapter, you can find a review of a large number of popular classic evolutionary methods for multimodal optimization, where a taxonomy of these methods into three classes is made for the first time. Methods are classified according to their implemented technique of having an explicit or implicit basin identification. Class A: (explicit basin identification), establishes a mapping from search space to basins. Whereas, class B: (basin avoidance or implicit basin identification) avoids searching in already explored locations. Class $\mathrm{C}$ : (diversity maintenance) is concerned with spreading the search over the whole search space without taking the search space topology into account.

Chapter 6 presents two NBC based optimization methods with their parameter settings (Niching Evolutionary Algorithm 1 and 2). Then, both NEA1 and NEA2 are evaluated on multimodal problems from the Black Box Optimization Benchmark (BOBB) set for the One-Global case and on the problem set of the IEEE CEC 2013 niching methods competition for All-Global case.

I found this book very beneficial and important as it presents a comprehensive discussion for evolutionary computation and optimization field with a focus on multimodal optimization. It provides an excellent explanation of the theoretical background of many topics in evolutionary computation, although it does not explain the details of many algorithms mentioned in the text. However, I really liked the huge number of references it has (252 references ranging from 1935 to 2013), which cover the research right from the start of the field.

I strongly recommend this book for graduate students or any researcher who wants to work in the EC field, rather than as a text book for an undergraduate course. I think it is very useful to understand the background of the main process of evolutionary computation algorithms and have this number of references. It also may help in improving some algorithms and may motivate the researcher to introduce new ones. A good aspect is that the chapters are self contained so that you can read individual chapters that you are interested in without the need to read the whole book. 